\title{
CONTRIBUCIÓN AL ESTUDIO FITOQUÍMICO DEL ZUMO DE Colignonia parviflora SUBSP. BIUMBELLATA (SAQTA)
}

\author{
Juan Carlos Chipayo Auccapuma, Jessica Ivana Nieto Juárez, \\ Ingrit Elida Collantes Díaz*
}

\begin{abstract}
RESUMEN
En el presente trabajo se investigó las raíces de Colignonia parviflora subsp. biumbellata, fue adquirida en la ciudad de Chinchero, a $3780 \mathrm{msnm}$. Fueron identificados 24 componentes químicos entre ésteres metílicos, monoterpeno, sesquiterpenos, fenilpropanos y alcoholes alifáticos en el aceite esencial de la raíz. La identificación fue a través de la comparación de los espectros de masas con el banco de datos del sistema de CG-EM (NIST 14) y por el análisis de sus espectros de masas obtenidos por cromatografía gaseosa acoplada a espectrómetro de masas. En la fase apolar del zumo de la raíz fueron identificados sus ácidos grasos mayoritarios y los metabolitos secundarios $\beta$-sitosterol, estigmasterol, estigmasta-7en-3-ol, estigmasta-7,22-dien-3-ol, el éster metílico del ácido ferúlico y el ácido oleánico.
\end{abstract}

Palabras clave: Colignonia parviflora subsp. biumbellata, saqta, aceite esencial, esteroides.

\section{CONTRIBUTION TO THE PHYTOCHEMICAL STUDY OF ZUMO FROM Colignonia parviflora SUBSP. BIUMBELLATA (SAQTA)}

\begin{abstract}
In the present work, the roots of Colignonia parviflora subsp. biumbellata, was acquired in the city of Chinchero at $3780 \mathrm{msnm}$. Twenty four chemical compounds were identified among methyl esters, monoterpenes, sesquiterpenes, phenylpropanoid and aliphatic alcohols in the root essential oil, the identification was through the comparasion of the mass spectra with the data bank of the CG-MS system (NIST 14) and by the analysis of their mass spectra obtained by gas chromatography coupled to a mass spectrometer. In the apolar phase of the root juice, its major fatty acids and secondary metabolites $\beta$-sitosterol, stigmasterol, stigmast7-en-3 $\beta$-ol, stigmast-7,22-dien-3 $\beta$-ol, methyl ester of ferulic acid and oleanic acid.
\end{abstract}

Key words: Colignonia parviflora subsp. biumbellata, saqta, essential oil, steroids.

Facultad de Ingeniería Química y Textil, Universidad Nacional de Ingeniería. Av. Túpac Amaru No 210, Rímac, Lima-Perú, ingrit_uni@hotmail.com 


\section{INTRODUCCIÓN}

La familia Nictaginaceae comprende cerca de 30 géneros y 400 especies, con distribución pantropical, principalmente en las regiones tropicales y subtropicales del nuevo mundo. La familia incluye arbustos, árboles y raramente lianas o hierbas. A través de los estudios moleculares se han determinado siete tribus que conforman la familia: Boldoeae, Bougainvilleae, Caribeeae, Colignonieae, Leucastereae, Nyctagineae y Pisonieae ${ }^{1}$. En el Perú se distribuyen 11 géneros con 53 especies, la mayoría son arbustos o hierbas². Dentro del género Colignonia están las especies C. glomerata Griseb, C. ovalifolia Heimerl, C. parviflora, C. pentoptera J.E. Bohlin, C. rufopilosa Kuntze, C. scandens Benth. La especie objetivo de nuestro estudio, Colignonia parviflora subsp. biumbellata es un arbusto que crece en la región andina del Perú, puede ser encontrada en el Cusco (Chinchero), Lima (Yauyos) ${ }^{3}$, La libertad ${ }^{4}$, Moquegua (Cuenca río Ilo-Moquegua) ${ }^{5}$, la especie es popularmente conocida con los nombres de "saqta", "sacha paraqai" y tradicionalmente los pobladores de la región de Chincheros de la provincia de Anta del departamento del Cusco, utilizan las ralladuras de la raíz en agua ya que forma bastante espuma que actúa como detergente y son usadas para el lavado de la lana de ovejas o alpaca para ser teñidas posteriormente, hecho que fue presenciado por los autores.

La especie en estudio no presenta estudios fitoquímicos, pero sí hay estudio de una especie próxima la $C$. scandens, se usa el decocto de la raíz para lavar la ropa y como depurativo de la piel en casos de sarampión, la planta es conocida con los nombres de sacha paracay, llupplunsha, jaboncillo y quinuanya. De C. scandens fueron aislados e identificados las siguientes saponinas chikusetsusaponin IV y chikusetsusaponin $\mathrm{V}^{6}$.

El presente trabajo reporta la identificación de 25 componentes químicos en el aceite esencial de la raíz de C. parviflora subsp. biumbellata, fueron identificados a través de la comparación de los espectros de masas con el banco de datos del sistema de CG-EM de NIST 14 y por análisis de sus espectros de masas obtenidos por cromatografía gaseosa acoplada a espectrómetro de masas. En la fase apolar del zumo de la raíz fueron identificados los ácidos grasos mayoritarios (linoleico, palmítico y 6-octadecenoico), así como también, fueron aislados e identificados los esteroides sitosterol (1), estigmasterol (2), estigmasta-7-en-3-ol (3) y estigmasta-7,22-dien-3-ol (4) también llamado de espinasterol, el fenil propanoide éster metílico del ácido ferúlico (5) y el ácido oleánico (6).

\section{PARTE EXPERIMENTAL}

\section{Colecta de la muestra e identificación botánica}

Fueron compradas las raíces (figura 1) en un mercado artesanal en Chinchero-Cusco (febrero de 2019) a $3780 \mathrm{msnm}$. La identificación botánica (tabla 1) fue realizada por el profesor Dr. Washington Galiano Sánchez de la Universidad Nacional de San Antonio de Abad del Cusco. 


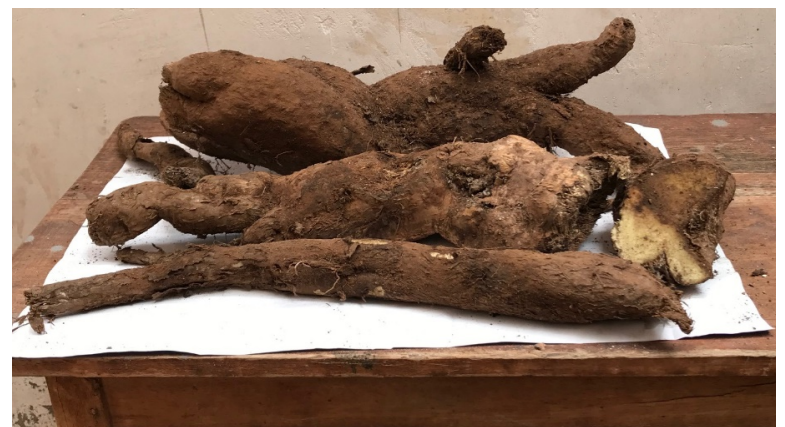

Figura 1. Raíces de saqta adquiridas en el mes de febrero de 2019.

Tabla 1. Identificación taxonómica de C. parviflora subsp. biumbellata (Ball) JEBohlin.

\begin{tabular}{ll}
\hline Clase: & Equisetopsida C. Agard \\
Subclase: & Magnoliidae Novák ex Takht. \\
Superorden: & Caryophyllanae Takht. \\
Orden: & Caryophyllales Juss. ex Bercht. \& J. Presl \\
Familia: & Nyctaginaceae Juss. \\
Género: & Colignonia Endl. \\
Especie: & Colignonia parviflora subsp. biumbellata (Ball) JEBohlin \\
\hline
\end{tabular}

\section{Extracción y aislamiento de los fitoconstituyentes}

Fueron adquiridos $2,547 \mathrm{~kg}$ de raíz fresca, la cual fue separada en dos partes, una parte $(850 \mathrm{~g})$ para la obtención del aceite esencial y la diferencia $(1,697 \mathrm{~kg})$ fue sometida a extracción del zumo (970 mL) (figura 2) en un extractor de jugos eléctrico doméstico de la marca OSTER, obteniéndose $727 \mathrm{~g}$ de bagazo de la raíz.

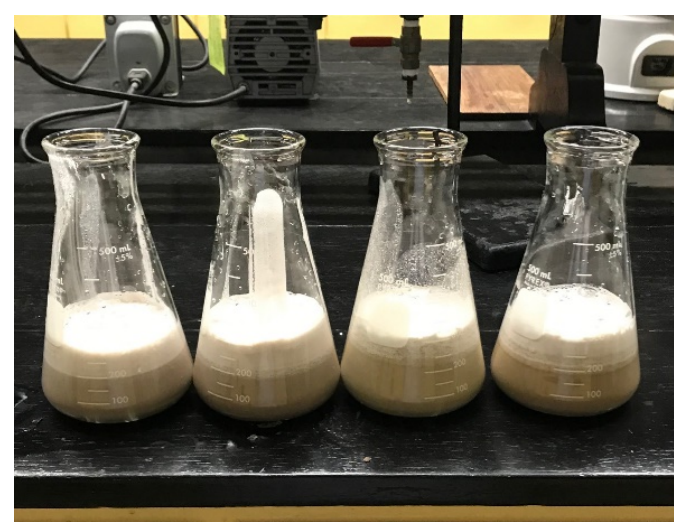

Figura 2. Zumo obtenido de la raíz saqta fresca. 
Obtención del aceite esencial.- Las raíces (850g) fueron sometidas a extracción del aceite esencial por hidrodestilación con aparato de Clevenger $^{7}$, el aceite esencial fue extraído con éter de petróleo y secado con sulfato de sodio anhidro para posteriormente ser analizado por cromatografía gaseosa acoplada a espectrómetro de masas (CG-EM) ${ }^{8}$.

Partición líquida-líquida del zumo.- El zumo fue sometido a partición líquido-líquido para la obtención de los residuos clorofórmico $(\mathrm{RCHCl} 3)$ 2,1535 g, residuo butanólico $(\mathrm{RBuOH})$ $3,172 \mathrm{~g}$ y residuo acuoso (RH2O) 12,34 g, las fases acuosas de los residuos fueron liofilizadas. El RCHCl3 fue sometido a fraccionamiento por cromatografía en columna abierta, usando como fase estacionaria sephadex LH-20 y los eluyentes fueron éter de petróleo (EP), diclorometano (DCM) y metanol $(\mathrm{MeOH})$, así se obtuvo la fracción de éter de petróleo (FEP) $1,125 \mathrm{~g}$, fracción de diclorometano (FDCM) 155,5 mg y la fracción metanólica (FMeOH) $0,873 \mathrm{~g}$.

Transesterificación de los ácidos grasos.- Ochenta miligramos de la FEP fue transesterificado para determinar el ácido graso mayoritario, siguiendo el método de obtención y análisis por Díaz et al., $2011^{10}$.

Fraccionamiento de la fracción de éter de petróleo y de diclorometano.- Las fracciones de éter de petróleo (FEP) y de diclorometano (FDCM) fueron sometidas a fraccionamiento por cromatografía en columna abierta por separado, usado como fase estacionaria sílica-gel (fase normal) y como eluyente la mezcla de los solventes (éter de petróleo, acetato de etilo, cloroformo y metanol) de polaridad creciente. Del fraccionamiento fueron obtenidos 68 y 34 subfracciones de la FEP y FDCM, respectivamente, las subfracciones fueron analizadas por separado por cromatografía de capa delgada analítica (usando como revelador $\mathrm{H} 2 \mathrm{SO} 4$ al $25 \%$, seguida de calentamiento) y reunidas siguiendo un patrón de similaridad. Después de ser analizados por separado se realizó la comparación final de las subfracciones de ambas columnas (FEP y FDCM) con la finalidad de obtener fracciones de mayores cantidades para realizar el aislamiento a través de cromatografía de capa delgada preparativa (placa de vidrio de $20 \times 20 \mathrm{~cm}$ ) usando como fase estacionaria sílica gel, las placas preparadas en el laboratorio tenían $1 \mathrm{~mm}$ de espesor. Para aislar las moléculas III, IV y VI se hizo un corte lateral $(\sim 1,5 \mathrm{~cm})$ en la placas preparativas y se reveló con $\mathrm{H}_{2} \mathrm{SO}_{4}$ al $25 \%$ ya que estas moléculas no revelan bajo la luz a 254 o $356 \mathrm{~nm}$ de una lámpara ultravioleta.

Los compuestos I y II son una mezcla y fueron obtenidos como cristales de la reunión de las fracciones 29-30 (FEP) con la fracción 16 (FDCM), estos cristales fueron sometidos a análisis por RMN para su identificación.

De la reunión de las muestras 38-40 (FEP) y 19-20 (FDCM) se obtuvo 18,3 mg de masa, esta muestra fue sometida a cromatografía de capa delgada preparativa (CCDP) con la mezcla de EP:AcOEt en la proporción 4:1 eluido por tres veces consecutivas, de la cual se obtuvo tres subfracciones, la subfracción 2 es la mezcla de los compuestos III y IV. 
La muestra 23-27 (FDCM) fue sometida a purificación a través de CCDP usando como eluente la mezcla de solventes EP:AcOEt 4:1, fue eluido por tres veces consecutivas, obteniéndose así el compuesto $\mathbf{V}$.

El compuesto VI fue obtenido de la purificación por cromatografía de capa delgada preparativa de la fracción 41- 44 (FEP) (25,7 mg) usando como eluente la mezcla de EP:AcOEt en la proporción 4:1 eluido por tres veces consecutivas.

\section{Análisis por cromatografía gaseosa acoplada a espectrómetro de masas}

Análisis de los ésteres metílicos.- El análisis por cromatografía gaseosa fue realizado en un cromatógrafo a gas acoplado a un espectrómetro de masas (Shimadzu, modelo CGMSQP2010 Ultra) con las siguientes condiciones de análisis: columna capilar Restek Rtx-5MS (30 $\mathrm{m} \times 0,25 \mathrm{~mm} \times 0,25 \mu \mathrm{m})$; temperatura del inyector $240^{\circ} \mathrm{C}$, temperatura del detector 230 ${ }^{\circ} \mathrm{C}$; impacto de electrones a $70 \mathrm{eV}$, con gas de arrastre helio a un flujo de $1,23 \mathrm{~mL} / \mathrm{min}$, con split $1 / 5$; con un programa de temperatura de $120^{\circ} \mathrm{C}\left(2^{\prime}\right)-280^{\circ} \mathrm{C}, 2^{\circ} \mathrm{C} /$ minuto; y con volumen de inyección de $3 \mu \mathrm{L}$.

La identificación de las sustancias componentes fue a través de la comparación de los espectros de masas con el banco de datos del sistema de CG-EM de NIST 14 lib. y con la inyección de los siguientes patrones: ésteres metílicos (Mixture for GC, 37 component fame, Lot: LRAC1814, Sigma-Aldrich).

Análisis de los aceites esenciales.- El análisis por cromatografía gaseosa fue realizado en un cromatógrafo a gas acoplado a un espectrómetro de masas (Shimadzu, modelo CGMSQP2010 Ultra) con las siguientes condiciones de análisis: columna capilar Restek Rtx-5MS (30 m x $0,25 \mathrm{~mm} \times 0,25 \mu \mathrm{m})$; temperatura del inyector $260{ }^{\circ} \mathrm{C}$, temperatura del detector $260{ }^{\circ} \mathrm{C}$; impacto de electrones a $70 \mathrm{eV}$, con gas de arrastre helio a un flujo de $1,48 \mathrm{~mL} / \mathrm{min}$, con split $1 / 5$; con un programa de temperatura de $80^{\circ} \mathrm{C}\left(5^{\prime}\right)-280^{\circ} \mathrm{C}\left(5^{\prime}\right), 8^{\circ} \mathrm{C} /$ minuto; y con volumen de inyección de $3 \mu \mathrm{L}$.

La identificación de las sustancias componentes fue a través de la comparación de los espectros de masas con el banco de datos del sistema de CG-EM de NIST 14 lib y por análisis de sus espectros de masas obtenidos por cromatografía gaseosa acoplada a espectrómetro de masas. Así como, con la inyección de los siguientes patrones: hidrocarburos (Mixture for GC, C8-C40 alkanes calibration, Lot LRAC1880 Sigma-Aldrich).

\section{Análisis por resonancia magnética nuclear}

Los espectros de resonancia magnética nuclear de ${ }^{1} \mathrm{H}$ y de ${ }^{13} \mathrm{C}$ fueron registrados en un espectrómetro Bruker de $500 \mathrm{MHz}$, operando a $500 \mathrm{MHz}$ para los espectros de hidrógeno y para la obtención de los espectros de carbono 13 operaba a $125 \mathrm{MHz}$. Los espectros fueron obtenidos en cloroformo y metanol deuterados $\left(\mathrm{CDC}_{13}\right.$ y $\left.\mathrm{CD}_{3} \mathrm{OD}\right)$ de la marca MERCK. 


\section{RESULTADOS Y DISCUSIÓN}

De la extracción por hidrodestilación se obtuvo $42 \mathrm{mg}$ de aceite esencial de la raíz de la saqta, representando ese valor el $0,0049 \%$ de rendimiento. El aceite inicialmente era transparente, con el pasar del tiempo se tornó de color amarillo claro. El análisis del aceite esencial por cromatografía gaseosa acoplada a espectrómetro de masas condujo a la identificación de 24 moléculas (tabla 2). Los compuestos mayoritarios son el $p$-vinil-guayacol (9,8 \%) y los ésteres metílicos de los ácidos grasos linoleico (28,6\%), linolénico (12,2\%), palmítico (5,9\%). También fueron identificados otros componentes como monoterpenos, fenilpropanos, sesquiterpenos, alcoholes alifáticos, éster metílico y éster butílico de ácido graso de bajos porcentajes.

Tabla 2. Composición química del aceite esencial de la raíz saqta, con su tiempo de retención y su porcentaje de presencia de cada componente.

\begin{tabular}{lllccccc}
\hline $\mathbf{N}^{\circ}$ & T. R. & $\mathbf{\%}$ & Nombre & $\mathbf{N}^{\circ}$ & $\mathbf{T} . \mathbf{R}$. & $\mathbf{\%}$ & Nombre \\
\hline $\mathbf{1}$ & 8857 & 0,53 & Nonanol & $\mathbf{1 3}$ & 15862 & 1,04 & cis-Sesquisabineno hidrato \\
$\mathbf{2}$ & 9342 & 0,25 & $\alpha$-Terpineol & $\mathbf{1 4}$ & 16419 & 1,93 & trans-Sesquisabineno hidrato \\
$\mathbf{3}$ & 10147 & 0,26 & Geraniol & $\mathbf{1 5}$ & 17282 & 0,45 & $\alpha$-Acorenol \\
$\mathbf{4}$ & 11894 & 9,77 & $p$-vinil-Guayacol & $\mathbf{1 6}$ & 17774 & 2,42 & $\beta$-Bisabolol \\
$\mathbf{5}$ & 13227 & 0,68 & $\beta$-Damascenona & $\mathbf{1 7}$ & 18440 & 0,15 & Éster metílico del ácido mirístico \\
$\mathbf{6}$ & 13542 & 0,20 & Metil eugenol & $\mathbf{1 8}$ & 18851 & 0,12 & Xantorrizol \\
$\mathbf{7}$ & 13784 & 1,24 & $\beta$-Ionol & $\mathbf{1 9}$ & 19602 & 1,47 & Salicilato de 2-etilhexil \\
$\mathbf{8}$ & 13886 & 0,18 & Cariofileno & $\mathbf{2 0}$ & 20495 & 0,33 & Hexadecanol \\
$\mathbf{9}$ & 14236 & 0,94 & Sesquisabineno & $\mathbf{2 1}$ & 21060 & 5,85 & Éster metílico de ácido palmítico \\
$\mathbf{1 0}$ & 14830 & 0,07 & $\gamma$-Curcumeno & $\mathbf{2 2}$ & 23092 & 28,64 & Éster metílico de ácido linoleico \\
$\mathbf{1 1}$ & 15557 & 1,01 & $\beta$-Sesquifelandreno & $\mathbf{2 3}$ & 23170 & 12,22 & Éster metílico del ácido linolénico \\
$\mathbf{1 2}$ & 15761 & 0,23 & Italiceno éter & $\mathbf{2 4}$ & 26011 & 1,68 & Éster butílico del ácido linolénico \\
& & & & & & \\
\hline
\end{tabular}

T.R.: Tiempo de retención.

Los ácidos grasos libres y los triglicéridos presentes en las fracciones apolares de los extractos vegetales no son volátiles, es por esa razón que no se pueden analizar por cromatografía gaseosa. Para identificar los ácidos grasos se realiza la transesterificación. El análisis de los ésteres metílicos presentes en la $\mathrm{FEP}$ del $\mathrm{RCHCl}_{3}$ del zumo de la raíz saqta fresca fue realizado por cromatografía gaseosa acoplando a espectrómetro de masas. En la tabla 3 se reporta la identificación de los tres ésteres metílicos mayoritarios que representan el 84,47\%, en la que se observa el ácido linoleico como mayoritario con 45,5\%, siendo este ácido considerado como un ácido graso esencial ${ }^{11}$.

Tabla 3. Porcentaje de la presencia de los ésteres metílicos mayoritarios en la fracción de éter de petróleo.

\begin{tabular}{cccl}
\hline $\mathbf{N}^{\circ}$ & T.R. & $\mathbf{\%}$ & \multicolumn{1}{c}{ Nombre } \\
\hline $\mathbf{1}$ & 29785 & 20,83 & Éster metílico del ácido palmítico \\
$\mathbf{2}$ & 37329 & 45,49 & Éster metílico del ácido linoleico \\
$\mathbf{3}$ & 37551 & 18,15 & Éster metílico del ácido 6-octadecenoico \\
\hline \multicolumn{5}{c}{ T.R. : Tiempo de retención. }
\end{tabular}


El fraccionamiento de las fracciones de éter de petróleo y de diclorometano del residuo clorofórmico del zumo de la raíz fresca de saqta condujo al aislamiento y/o identificación de 6 metabolitos secundarios (figura 3) a través del análisis de sus espectros de RMN de $1 \mathrm{H}$ y RMN de ${ }^{13} \mathrm{C}$. Los compuestos 1-2, y 3-4 fueron obtenidos en mezcla, ambas mezclas son dos pares de esteroides muy comunes como constituyentes químicos en las plantas, pueden estar en diferentes proporciones y son inseparables, por ejemplo tenemos la mezcla de $\mathbf{1}$ y $\mathbf{2}$, fueron obtenidos de Cochlospermum vitifolium y en la proporción de 3:1 ${ }^{12}$.

Compuestos 1 y 2.- Fue obtenido $37 \mathrm{mg}$ de unos cristales transparentes. A través del análisis de sus espectros de $\mathrm{RMN}-{ }^{13} \mathrm{C}$ (tabla 4 ) y $\mathrm{RMN}-{ }^{1} \mathrm{H}$ fue determinado que el compuesto 1 es el esteroide $\beta$-sitosterol y el compuesto 2 es el estigmasterol. Por la integración de sus señales del espectro de $\mathrm{RMN}-{ }^{1} \mathrm{H}$ podemos afirmar que los compuestos $\mathbf{1}$ y $\mathbf{2}$ se encuentran en la relación de 1:3. Los desplazamientos químicos del compuesto $1\left(\mathrm{CDCl}_{3}, 500 \mathrm{MHz}\right) \delta$ ppm: 0,69 (3H, s, H-18), 0,81 (3H, d, $J=6,7 \mathrm{~Hz}, \mathrm{H}-26), 0,83$ (3H, d, $J=6,7 \mathrm{~Hz}, \mathrm{H}-27), 0,88$ (3H, t, $J=6 \mathrm{~Hz}, \mathrm{H}-29), 0,93$ (3H, d, $J=6,4 \mathrm{~Hz}, \mathrm{H}-21), 1,02$ (3H, s, H-19), 1,24 (5H, m, H-11, 22, $23,1,8), 1,31$ (5H, m, H-1, 15, 16, 12, 2), 1,42 (2H, m, H-9, 14), 1,51 (6H, m, H-2, 12, 11, 28, 24, 17), 1,68 (4H, m, H-7, 20, 15, 16), 1,84 (1H, m, H-25), 2,01 (1H, m, H-4ax), 2,26 (1H, m, $\mathrm{H}-4 \mathrm{eq}), 3,52(1 \mathrm{H}, \mathrm{m}, \mathrm{H}-3), 5,35(1 \mathrm{H}, \mathrm{m}, \mathrm{H}-6)^{13}$. Del compuesto $2(\mathrm{CDCl} 3,500 \mathrm{MHz}) \delta \mathrm{ppm}$ : $0,71$ (3H, s, H-18), 0,81 (3H, d, $J=6,7 \mathrm{~Hz}, \mathrm{H}-26), 0,82$ (3H, d, $J=6,7 \mathrm{~Hz}, \mathrm{H}-27), 0,83$ (3H, t, $J=6 \mathrm{~Hz}, \mathrm{H}-29), 0,90$ (3H, d, $J=6,7 \mathrm{~Hz}, \mathrm{H}-21), 1,38$ (3H, s, H-19), 3,53 (1H, m, H-3), 5,03 $(1 \mathrm{H}, \mathrm{dd}, J=15,3,8,5 \mathrm{~Hz}, \mathrm{H}-23), 5,15$ (1H, dd, $J=15,3,8,55 \mathrm{~Hz}, \mathrm{H}-22), 5,35(1 \mathrm{H}, \mathrm{m}, \mathrm{H}-6)^{14}$.

Compuesto 3 y 4.- Fue obtenido 9,4 mg, esta muestra se presenta como un sólido blanco. Fue identificada como una mezcla de estigmasta-7-en-3-ol (3) y estigmasta-7,22-dien-3-ol (4) por análisis de sus desplazamientos químicos de sus espectros de $\mathrm{RMN}-{ }^{13} \mathrm{C}$ (tabla 4) y RMN-1H y por integración de sus señales se puede justificar que están en la relación de 1:7, respectivamente. Compuesto $3\left(\mathrm{CDCl}_{3}, 500 \mathrm{MHz}\right) \delta \mathrm{ppm}: 0,55(3 \mathrm{H}, \mathrm{s}, \mathrm{H}-18), 0,80(3 \mathrm{H}, \mathrm{s}$, H-19), 0,81 (3H, d, $J=6,1 \mathrm{~Hz}, \mathrm{H}-27), 0,83(3 \mathrm{H}, \mathrm{d}, J=6,1 \mathrm{~Hz}, \mathrm{H}-26), 0,86(3 \mathrm{H}, \mathrm{t}, J=7,63$ Hz, H-29), 0,94 (3H, d, $J=6,7$ Hz, H-21), 3,61 (1H, m, H-3), 5,17 (1H, m, H-7). Compuesto $4\left(\mathrm{CDCl}_{3}, 500 \mathrm{MHz}\right) \delta \mathrm{ppm}: 0,56(3 \mathrm{H}, \mathrm{s}, \mathrm{H}-18), 0,81(3 \mathrm{H}, \mathrm{s}, \mathrm{H}-19), 0,81(3 \mathrm{H}, \mathrm{d}, J=6,1 \mathrm{~Hz}$, $\mathrm{H}-23), 0,82(3 \mathrm{H}, \mathrm{t}, J=7,35 \mathrm{~Hz}, \mathrm{H}-29), 1,04(3 \mathrm{H}, \mathrm{d}, J=6,72 \mathrm{~Hz}, \mathrm{H}-21), 3,6(1 \mathrm{H}, \mathrm{m}, \mathrm{H}-3)$, $5,04$ (1H, dd, $J=8,55,15,25 \mathrm{~Hz}, \mathrm{H}-23), 5,15$ (1H, m, H-7), 5,17 (1H, dd, $J=8,55,14,95$ $\mathrm{Hz}, \mathrm{H}-22) 15$.

Compuestos 5.- Fue obtenido 7,2 mg de un sólido de color amarillo claro, fue identificado como el éster metílico del ácido ferúlico a través del análisis de sus desplazamientos químicos de sus espectros de $\mathrm{RMN}-{ }^{13} \mathrm{C}$ (tabla 4$)$ y $\mathrm{RMN}-{ }^{1} \mathrm{H}\left(\mathrm{CDCl}_{3}, 500 \mathrm{MHz}\right) \delta \mathrm{ppm}: 3,81(3 \mathrm{H}$, s, H-1" '), 3,94 (3H, s, 3-OMe), 6,29 (1H, d, $J=15,9 \mathrm{~Hz}, \mathrm{H}-2$ '), 6,93 (1H, d, $J=8,25 \mathrm{~Hz}, \mathrm{H}-5)$, 7,04 (1H, d, $J=2,1 \mathrm{~Hz}, \mathrm{H}-2), 7,08(1 \mathrm{H}, \mathrm{dd}, J=8,5,1,55 \mathrm{~Hz}, \mathrm{H}-6), 7,63(1 \mathrm{H}, \mathrm{d}, J=16,15$ $\left.\mathrm{Hz}, \mathrm{H}-1^{\prime}\right)^{16}$. 


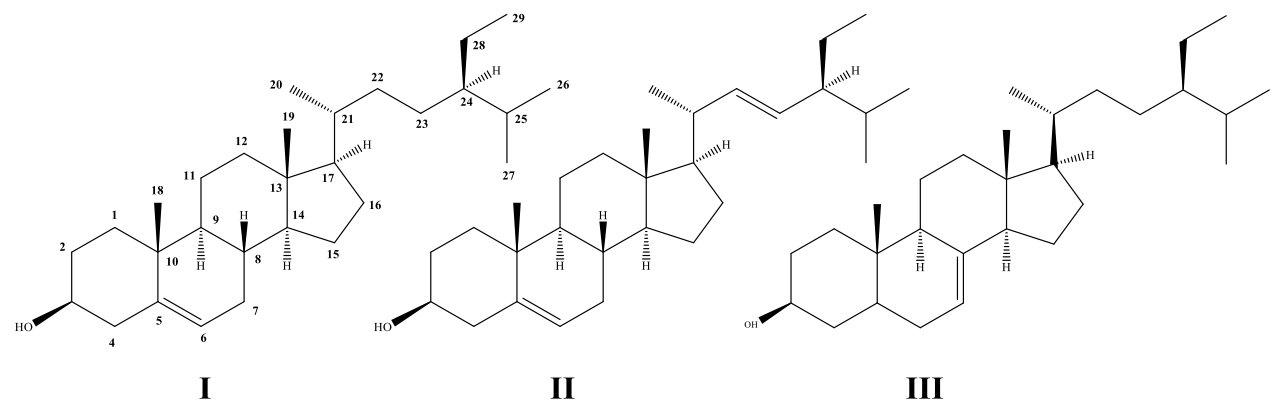

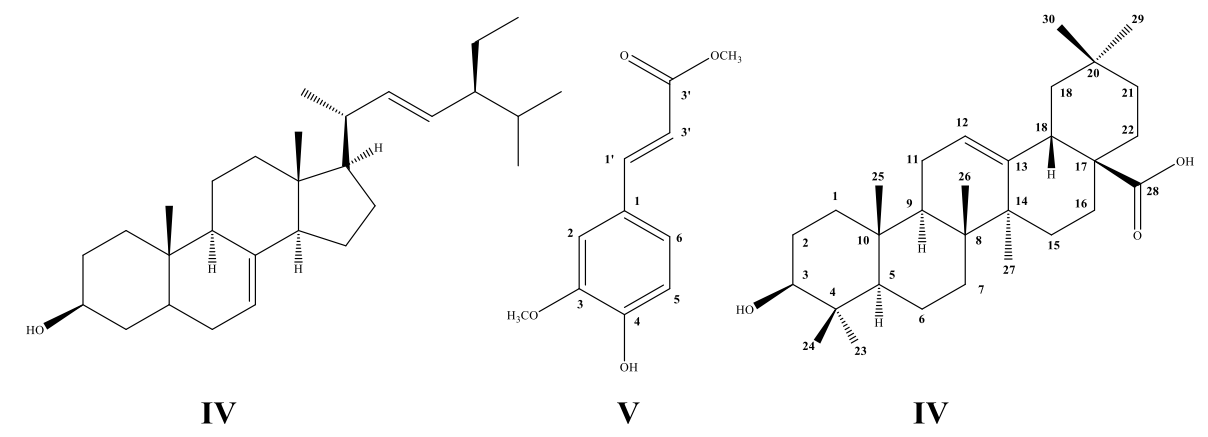

Figura 3. Moléculas identificadas en el zumo de la raíz de C. parviflora subsp. biumbellata. (sitosterol 1, estigmasterol 2, estigmasta-7-en-3-ol 3, estigmasta-7,22dien-3-ol 4, éster metílico del ácido ferúlico 5 y ácido oleánico 6).

Compuesto 6.- Se obtuvo $12 \mathrm{mg}$ de un polvo crema. Fue identificado como ácido oleánico por análisis de sus desplazamientos químicos de sus espectros de $\mathrm{RMN}-{ }^{13} \mathrm{C}$ (tabla 4) y RMN${ }^{1} \mathrm{H}\left(\mathrm{CD}_{3} \mathrm{OD}, 500 \mathrm{MHz}\right) \delta$ ppm: 0,78 (3H, s, H-25), 0,83 (3H, s, H-29), 0,91 (6H, s, H-24 y 30), 0,95 (3H, s, H-26), 0,98 (3H, s, H-23), 1,16 (3H, s, H-27), 2,85 (1H, dd, J=13,7, 4 Hz, $\mathrm{H}-18), 3,15$ (1H, dd, $J=11,6,4,9 \mathrm{~Hz}, \mathrm{H}-3), 5,24(1 \mathrm{H}, \mathrm{dd}, J=3,7,3,35 \mathrm{~Hz}, \mathrm{H}-12)^{17}$. 
Tabla 4. Desplazamiento químico del $\mathrm{RMN}$ de ${ }^{13} \mathrm{C}(\delta \mathrm{ppm}, 125 \mathrm{MHz})$ de las moléculas identificadas en el zumo de la raíz de C. parviflora subsp. biumbellata.

\begin{tabular}{|c|c|c|c|c|c|c|c|}
\hline C & 1 & 2 & 3 & 4 & 6 & C & 5 \\
\hline & & \multicolumn{2}{|c|}{$\mathrm{CDCl}_{3}$} & & $\mathrm{CD}_{3} \mathrm{OD}$ & & $\mathrm{CDCl}_{3}$ \\
\hline 1 & 37,28 & 37,28 & 37,17 & 31,17 & 38,34 & 1 & 126,99 \\
\hline 2 & 31,68 & 31,88 & 31,52 & 31,52 & 26,56 & 2 & 109,36 \\
\hline 3 & 71,84 & 71,84 & 71,08 & 71,08 & 79,89 & 3 & 146,75 \\
\hline 4 & 42,24 & 42,32 & 37,17 & 38,03 & 38,34 & 4 & 147,97 \\
\hline 5 & 140,77 & 140,77 & 40,29 & 40,29 & 56,94 & 5 & 114,71 \\
\hline 6 & 121,72 & 121,72 & 29,66 & 29,66 & 19,68 & 6 & 123,03 \\
\hline 7 & 31,93 & 31,93 & 117,47 & 117,47 & 33,22 & 1 , & 144,92 \\
\hline 8 & 31,93 & 31,93 & 139,58 & 139,58 & 40,00 & 2' & 115,21 \\
\hline 9 & 50,16 & 50,25 & 49,48 & 49,48 & 47,94 & 3' & 167,68 \\
\hline 10 & 36,53 & 36,53 & 34,25 & 34,25 & 37,08 & $1 "$ & 55,94 \\
\hline 11 & 21,07 & 21,04 & 21,57 & 21,57 & 24,17 & 30Ме & 55,94 \\
\hline 12 & 39,80 & 39,71 & 39,48 & 39,48 & 123,68 & & \\
\hline 13 & 42,24 & 42,32 & 43,29 & 43,29 & 145,55 & & \\
\hline 14 & 56,79 & 56,88 & 55,06 & 55,14 & 42,99 & & \\
\hline 15 & 24,32 & 24,38 & 23,02 & 23,02 & 28,04 & & \\
\hline 16 & 28,26 & 28,91 & 27,97 & 24,49 & 24,69 & & \\
\hline 17 & 56,09 & 55,99 & 56,12 & 55,94 & 47,52 & & \\
\hline 18 & 11,86 & 11,99 & 11,85 & 12,05 & 43,08 & & \\
\hline 19 & 19,39 & 19,39 & 13,04 & 13,04 & 47,52 & & \\
\hline 20 & 36,53 & 40,47 & 36,59 & 40,81 & 31,78 & & \\
\hline 21 & 18,79 & 21,22 & 18,99 & 21,38 & 34,06 & & \\
\hline 22 & 33,59 & 138,31 & 33,93 & 138,16 & 33,76 & & \\
\hline 23 & 26,12 & 129,30 & 27,19 & 129,48 & 28,91 & & \\
\hline 24 & 46,03 & 45,87 & 45,87 & 51,26 & 16,45 & & \\
\hline 25 & 29,14 & 33,97 & 29,20 & 31,88 & 16,04 & & \\
\hline 26 & 19,81 & 18,98 & 19,8 & 21,07 & 17,94 & & \\
\hline 27 & 19,39 & 21,21 & 18,99 & 18,99 & 26,56 & & \\
\hline 28 & 22,69 & 25,40 & 23,02 & 25,4 & 181,33 & & \\
\hline 29 & 12,25 & 12,05 & 12,05 & 12,25 & 34,21 & & \\
\hline 30 & - & - & - & - & 24,29 & & \\
\hline
\end{tabular}

\section{CONCLUSIONES}

El estudio fitoquímico de las raíces de Colignonia parviflora subsp. biumbellata condujo a la identificación de 25 compuestos químicos en el aceite esencial, 3 ésteres metílicos en la fase apolar del zumo de la raíz y 6 metabolitos secundarios, que son compuestos ya identificados en otras plantas como lo demostramos al comparar los desplazamientos químicos de los metabolitos con la literatura correspondiente. Asimismo, los resultados obtenidos con este trabajo son por primera vez reportados para C. parviflora subsp. biumbellata. 


\section{AGRADECIMIENTOS}

Los autores agradecen al Instituto de Investigación de la Facultad de Ingeniería Química y Textil (II-FIQT) de la Universidad Nacional de Ingeniería (UNI) por el apoyo económico brindado para la realización de este trabajo de investigación (Proyecto de Investigación Formativa 2019 código: CPDI1-2019-2.

\section{REFERENCIAS BIBLIOGRÁFICAS}

1. Giulietti AM, Nogueira MGC. Flora das cangas da Serra dos Carajás, Pará, Brasil: Nyctaginaceae. Rodriguésia. 2017; 68(3): 1045 - 1051.

2. León B. Nyctaginaceae endémicas del Perú. Rev Perú Biol. 2006; 13(2): 469s - 470s.

3. Beltrán H. Catálogo de flora vascular del distrito de Laraos (Yauyos, Lima). Arnaldoa. 2018; 25(2): 565 - 596.

4. Aredo V, Carranza-Cabrera J, Siche R. Inventario de especies vegetales de la Libertad (Perú) y análisis de su potencial agroindustrial. Agroindustrial Science. 2017; 7(2): 87104.

5. Ministerio del Ambiente Perú. Indicadores Ambientales Moquegua. Díaz VM, Chambe HP. Primera edición. Lima: Serie Indicadores Ambientales. 2013; 252pp.

6. De Feo V, Paciente S, Pizza C, Soria RU. Saponins from Colignonia scandens Benth. (Nyctaginaceae). Biochem Syst Ecol. 1998; 26(2): 251 - 253.

7. Ministerio de Saude. Farmacopeia brasileira, 4 ed. Parte II, fascículo 2. In Diario Oficial, Suplemento $\mathrm{N}^{\circ} 1,2001$.

8. De Oliveira TS, Bombo AB, De Oliveira ASS, Garcia VL, Appezzato-da-Glória B. Seasonal variation of the essential oil from two Brazilian native Aldama La Llave (Asteraceae) species. An Acad Bras Ciênc. 2016; 88(3 Suppl.): 1899 - 1907.

9. Gusmão DF, Estork DM, Paciencia MLB, Díaz IEC, Frana SA, Rodrigues PA, et al. Preliminary evaluation of the acute toxicity related to Abarema auriculata to mice and investigation of cytotoxicity of isolated flavonones. PharmacologyOnline. 2013; 30(1): $113-127$.

10. Díaz IEC, Gonçalves EG, Marques MOM, Yoshida M. Incidencia de ácidos grasos en especies de Araceas. Rev Soc Quím Perú. 2011; 77(4): 275 - 284.

11. Moretto E, Fett R. Tecnologia de óleos e gorduras vegetais na indústria de alimentos. Primeira edição. São Paulo, Brasil: Valera editora e livraria Ltda; 1998, 150pp.

12. De Almeida SCX, De Lemos TLG, Silveira ER, Pessoa ODL. Constituintes químicos voláteis e não voláteis de Cochlospermum vitifolium (Willdenow) Sprengel. Quím Nova. 2005; 28(1): $57-60$.

13. Abdullah NH, Salim F, Ahmad R. Chemical constituents of Malaysian U. cordata var. ferruginea and their in vitro $\alpha$-glucosidase inhibitory activities. Molecules. 2016; 21(5): 525.

14. Chaturvedula VSP, Prakash I. Isolation of stigmasterol and $\beta$-sitosterol from the dichloromethane extract of Rubus suavissimus. Int Curr Pharm J. 2012; 1(9): 239 - 242. 
15. Kojima H, Sato N, Hatano A, Ogura H. Sterol glucosides from Prunella vulgaris Phytochem. 1990; 29(7): 2351-2355.

16. Li N-G, Shi Z-H, Tang Y-P, Li B-Q, Duan J-A. Highly efficient esterification of ferulic acid under microwave irradiation. Molecules. 2009; 14(6): 2118 - 2126.

17. De Sousa LRF, Santos MHF, Severino VGP, Severino RP, Viera PC. New degraded quinone diterpenoid from the stems of Byrsonima coccolobifolia Kunth (Malpighiaceae) Quím Nova 2018; 41(2): 184 - 188. 